\title{
Protocol for a randomized controlled trial of perioperative S-1 plus oxaliplatin combined with apatinib and camrelizumab in patients with resectable, locally advanced gastric or gastroesophageal junction adenocarcinoma
}

\author{
Yanan Zheng ${ }^{1}$, Zhenqiang Wang ${ }^{1}$, Chao Yan ${ }^{1}$, Min Yan ${ }^{1}$, Zhiguo Hou ${ }^{2}$, Rongrong Zheng ${ }^{2}$, Zhenggang Zhu ${ }^{1}$, \\ Chen $\mathrm{Li}^{1}$ \\ ${ }^{1}$ Department of Gastrointestinal Surgery, Ruijin Hospital Affiliated to Shanghai Jiao Tong University School of Medicine, Shanghai Institute of \\ Digestive Surgery, Shanghai Key Laboratory of Gastric Neoplasms, Shanghai, China; ${ }^{2}$ Jiangsu Hengrui Pharmaceuticals Co., Ltd., Shanghai, China \\ Correspondence to: Zhenggang Zhu; Chen Li. Department of Gastrointestinal Surgery, Ruijin Hospital Affiliated to Shanghai Jiao Tong University \\ School of Medicine, Shanghai Institute of Digestive Surgery, Shanghai Key Laboratory of Gastric Neoplasms, No. 197 Ruijin ER Road, Huangpu \\ District, Shanghai 200025, China. Email: zzg1954@hotmail.com; leedoctor@sina.com.
}

Background: Perioperative therapy can improve the low survival benefit of surgery alone for locally advanced gastric cancer. The introduction of immunotherapy and its combination with chemotherapy and/ or targeted therapy has created more opportunities for optimal treatment. The aim of the present study was to compare the efficacy and safety of S-1 plus oxaliplatin (SOX) combined with apatinib (SOXA) or SOX combined with apatinib and camrelizumab (SOXAP) versus SOX as the perioperative therapy for resectable, locally advanced gastric or gastroesophageal junction (GEJ) adenocarcinoma.

Methods: The study was a multicenter, randomized, open-label, parallel-controlled trial conducted in China. Eligible participants were randomized to the SOX, SOXA, and SOXAP groups. Patients received three pre-operative and three postoperative 3-week cycles of SOX or SOXA or SOXAP, followed by apatinib (SOXA group) or apatinib combined with camrelizumab (SOXAP group) for 3 cycles, which could be continued at the investigator's choice. Overall treatment is up to 1 year of apatinib and up to 17 cycles of camrelizumab. SOX is $130 \mathrm{mg} / \mathrm{m}^{2}$ oxaliplatin on day 1 plus S-1 orally twice daily on days 1 to 14 . Apatinib is orally administered at a dose of $500 \mathrm{mg}$ (SOXA group) or $250 \mathrm{mg}$ (SOXAP group) on days 1 to 21, and camrelizumab $200 \mathrm{mg}$ is given intravenously once every 3 weeks. The primary endpoint was major pathological response assessed by blinded independent review committee. The secondary endpoints included pathological complete response, lymph node status after neoadjuvant therapy, margin-free resection rate, progression-free survival (PFS), disease-free survival (DFS), overall survival (OS), and safety.

Discussion: The trial provides important data regarding the use of perioperative SOXAP and SOXA for patients with resectable, locally advanced gastric or GEJ adenocarcinoma. The results will contribute to optimal perioperative disease treatment.

Trial registration: ClinicalTrials.gov (no. NCT04208347). First posted on December 23, 2019.

Keywords: Gastric cancer; S-1 plus oxaliplatin (SOX); apatinib; camrelizumab; perioperative therapy

Submitted Jul 04, 2020. Accepted for publication Dec 16, 2020.

doi: $10.21037 /$ atm-20-7802

View this article at: http://dx.doi.org/10.21037/atm-20-7802 


\section{Introduction}

Gastric cancer is one of the most common cancers worldwide, and $42.6 \%$ patients with newly diagnosed gastric cancer are from China (1). Surgery is the most effective treatment for gastric cancer. However, the survival benefit of surgery alone is inadequate in a large number of patients with resectable disease due to advanced stage. The 3-year overall survival (OS) rate is $<50 \%$ in Chinese patients with stage III gastric cancer after surgery (2). The application of perioperative chemoradiotherapy may shrink the tumor and eradicate the micrometastases, leading to an increased margin-free (R0) resection rate, fewer recurrences and metastases, and improved survival. The MAGIC and FNCLCC/FFCD trials confirmed the efficacy and safety of perioperative chemotherapy in patients with resectable gastric cancer $(3,4)$, which has been recommended in various guidelines $(5,6)$. The RESOLVE trial compared perioperative chemotherapy of S-1 plus oxaliplatin (SOX) versus SOX or capecitabine plus oxaliplatin (XELOX) as a postoperative chemotherapy in Chinese patients with locally advanced gastric or gastroesophageal junction (GEJ) adenocarcinoma. The primary endpoint, 3-year disease-free survival (DFS) rate was significantly higher with perioperative SOX than with postoperative XELOX (62\% vs. 54.8\%), suggesting perioperative SOX as a novel standard regimen for locally advanced gastric or GEJ adenocarcinoma (7). A recent phase II trial of perioperative SOX plus preoperative apatinib, a vascular endothelial growth factor (VEGF) receptor tyrosine kinase inhibitor, showed favorable antitumor activity and manageable toxicity in patients with locally advanced gastric cancer (8), indicating the value of anti-angiogenic agents in the perioperative setting.

The introduction of immunotherapy and its combination with chemotherapy and/or targeted therapy has created more opportunities for the optimal treatment of cancer. In recent years, numerous studies have demonstrated the mechanisms and therapeutic effects of immune checkpoint inhibitors in combination with conventional chemotherapy or targeted therapy for a variety of cancers (9-13), including gastric cancer (14,15). Camrelizumab (SHR-1210), a novel programed cell death protein 1 antibody, has shown promising efficacy in patients with recurrent or metastatic gastric or GEJ cancer (16). A recent phase II trial suggested that camrelizumab combined with XELOX, followed by camrelizumab combined with apatinib, was well tolerated and generated a good response as the first-line therapy for advanced or metastatic gastric or GEJ cancers (17). Many trials of neoadjuvant immunotherapy, including camrelizumab, combined with other perioperative therapy, are ongoing to establish an optimal perioperative strategy.

The aim of the present study was to compare the efficacy and safety of SOX combined with apatinib (SOXA) or SOX combined with apatinib and camrelizumab (SOXAP) versus SOX as the perioperative therapy for resectable, locally advanced gastric or GEJ adenocarcinoma.

We present the protocol in accordance with the SPIRIT reporting checklist (available at http://dx.doi.org/10.21037/ atm-20-7802).

\section{Methods}

\section{Study design}

The present study was a multicenter, randomized, openlabel, parallel-controlled trial conducted in China (NCT04208347). The study flow is displayed in Figure 1.

\section{Inclusion criteria}

The present study is performed in accordance with the Declaration of Helsinki (as revised in 2013), Good Clinical Practice, and related laws. The protocol has been approved by the Ethics Committee at Ruijin Hospital affiliated with the School of Medicine at Shanghai Jiao Tong University (2019), (clinical ethics approval No. 212-3). All study participants provided signed informed consent prior to study commencement. The inclusion criteria were: (I) age 18-75 years; (II) pathologically proven gastric or GEJ adenocarcinoma; (III) resectable cancer with clinical stage cT3/4aN+M0 confirmed by computed tomography (CT) and or magnetic resonance imaging (MRI), according to American Joint Committee on Cancer (AJCC) criteria, 8th edition (18); (IV) no prior cancer treatment; (V) intention to have surgery after completing neoadjuvant therapy; (VI) ability to swallow tablets; (VII) Eastern Cooperative Oncology Group performance status of 0-1; (VIII) life expectancy $\geq 12$ months; and (IX) adequate organ functions, as follows: absolute neutrophil count $\geq 1.5 \times 10^{9} / \mathrm{L}$, platelets $\geq 80 \times 10^{9} / \mathrm{L}$, hemoglobin $\geq 80 \mathrm{~g} / \mathrm{L}$ (did not receive blood transfusion or blood products, and granulocytecolony stimulating factors or other hematopoietic stimulating factors within the past 14 days); total bilirubin $<1.5 \times$ the upper limit of normal (ULN) and aspartate aminotransferase and/or alanine transaminase $\leq 2.5 \times \mathrm{ULN}$; 


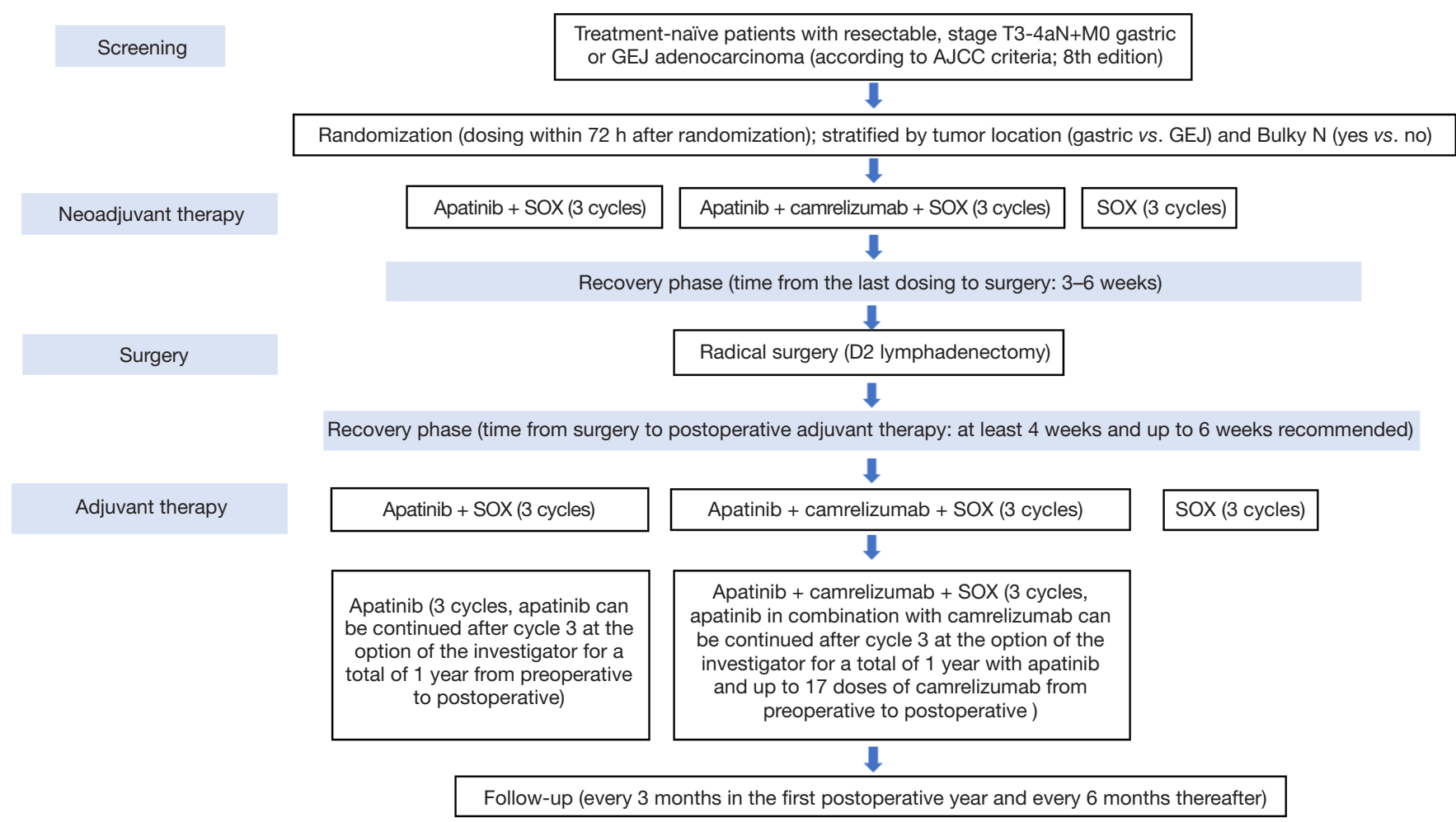

Figure 1 Study flowchart. AJCC, American Joint Committee on Cancer; GEJ, gastroesophageal junction; SOX, S-1 plus oxaliplatin.

and serum creatinine $\leq 1.5 \times$ ULN or endogenous creatinine clearance rate $>50 \mathrm{~mL} /$ minute.

Female participants of reproductive age took a serum pregnancy test within 72 hours prior to the first dose. Those with a negative result were included, and the women consented to using contraception during the study and within 120 days after the last dose. Male participants with partners of reproductive age underwent surgical sterilization or consented to using contraception during the study and within 120 days after the last dose.

\section{Exclusion criteria}

Exclusion criteria were (I) human epidermal growth factor receptor-2 positive; (II) gastric proximal GEJ cancer, and distance from the tumor center to GEJ $\leq 2 \mathrm{~cm}$; (III) peritoneal metastasis, positive peritoneal cytology results (CY1P0), or T4b according to the AJCC criteria, 8th edition (18); (IV) presence of unresectable factors, including tumours, or contraindications to surgery, or refusal to undergo surgery; $(\mathrm{V})$ previous or other co-existing malignant tumors, except for cured cutaneous basal cell carcinoma, cervical carcinoma in situ, or breast carcinoma in situ; (VI) uncontrolled hypertension (systolic blood pressure $\geq 140 \mathrm{mmHg}$ or diastolic blood pressure $\geq 90 \mathrm{mmHg}$ ); (VII) clinical manifestations or diseases of the heart: New York Heart Association grade $\geq 2$ heart failure or color Doppler echocardiography showing left ventricular ejection fraction $<50 \%$; unstable angina pectoris; myocardial infarction within 1 year; resting electrocardiogram (ECG) showing corrected QT interval (QTc) $>450 \mathrm{~ms}$ in males or $>470 \mathrm{~ms}$ in females; resting ECG showing abnormalities of important clinical significances (i.e., abnormalities in heart rate, conduction, and morphologic characteristics), complete left bundle branch block, grade 2-3 heart block, or PR interval $>250 \mathrm{~ms}$; and having factors that can increase the risks of QTc prolongation or heart rate abnormalities (i.e., heart failure, hypokalemia, congenital long QT syndrome, family history of long QT syndrome, QT prolonging drugs, or direct relative having died of unknown reasons < 40 years of age); (VIII) history of gastrointestinal perforation, abdominal abscess, or intestinal obstruction (within 3 months), or imaging examinations and/or clinical manifestations suggesting current intestinal obstruction; (IX) coagulation disorders (international normalized ratio $>2.0$ or prothrombin time $>16$ seconds), bleeding tendency, 
or currently receiving thrombolysis or anticoagulation therapy (prophylactic use of low-dose aspirin or lowmolecular heparin is acceptable); (X) clinically significant bleeding symptoms or evident bleeding tendency within 3 months before randomization (i.e., gastrointestinal bleeding, hemorrhagic gastric ulcer, or vasculitis); (XI) arterial/venous thrombus within 6 months before randomization, such as cerebrovascular accident (including transient ischemic attack, cerebral hemorrhage, and cerebral infarction), deep venous thrombosis, or pulmonary embolism; (XII) hereditary or acquired bleeding or thrombosis tendency, such as hemophilia, coagulation disorders, or thrombocytopenia; (XIII) active ulcer, unhealed trauma, or bone fracture; (XIV) urine routine examinations suggesting urine protein $\geq++$ and 24 -hour proteinuria $>1.0 \mathrm{~g}$; (XV) active infection requiring antimicrobial treatment; (XVI) active hepatitis; (XVII) congenital or acquired immunodeficiency; (XVIII) history or planned organ transplantation or allogeneic bone marrow transplantation; (XIX) current interstitial pneumonia or interstitial lung disease; history of interstitial pneumonia or interstitial lung disease requiring steroid treatment; pulmonary fibrosis, cryptogenic organizing pneumonia (i.e., obliterative bronchiolitis), pneumoconiosis, drugrelated pneumonia, or idiopathic pneumonia that can influence the assessment and treatment of immunerelated pulmonary toxicity; CT showing active pneumonia or severe pulmonary dysfunction at screening; or active pulmonary tuberculosis; and $(\mathrm{XX})$ any active autoimmune disease or history of autoimmune disease with relapse risk [including autoimmune hepatitis, interstitial pneumonia, uveitis, enteritis, hypophysitis, vasculitis, nephritis, hyperthyroidism, and hypothyroidism (except for participants for whom the disease can be controlled by hormone replacement therapy)]; participants with skin diseases requiring systemic treatments (i.e., leukoderma, psoriasis, and hair loss) or type I diabetes mellitus not controlled by insulin treatment, or a history of asthma that requires intervention; asthma participants requiring bronchodilators; (XXI) taking immunosuppressive drugs or systemic corticosteroid treatment within 7 days before randomization (>10 $\mathrm{mg} /$ day prednisone or other equivalent drugs); (XXII) receiving attenuated live vaccine within 28 days before randomization or intending to receive attenuated live vaccine during the treatment period or within 60 days after the last dose; (XXIII) using potent CYP3A4 inducer within 2 weeks or potent CYP3A4 inhibitor within 1 week before randomization; (XXIV) therapeutic antibiotics administered orally or intravenously for 4 weeks prior to randomization (except prophylactic antibiotics administered intravenously for less than 48 hours); (XXV) allergic to any study drug or ingredient; (XXVI) participating in other clinical trials within 4 weeks before randomization; (XXVII) lactating women; and (XXVIII) any other factors that can influence the study results or lead to study termination, as deemed by the investigators.

\section{Randomization and treatment}

Eligible participants were randomized to the SOX, SOXA, and SOXAP groups. The participants were stratified by tumor location (GEJ vs. gastric) and bulky $\mathrm{N}$ (yes $v s$. no). Bulky $\mathrm{N}$ was defined as at least two adjacent lymph nodes along the celiac artery, splenic artery, common hepatic artery or proper hepatic artery $\geq 1.5 \mathrm{~cm}$ or one such lymph node $\geq 3 \mathrm{~cm}$. Surgery was conducted 3-6 weeks after the last dose of neoadjuvant therapy, and the postoperative adjuvant therapy was conducted at least 4 weeks later, with a maximum recommended interval of 6 weeks.

The SOX group underwent preoperative SOX treatment (3 cycles) + surgery + postoperative SOX treatment (3 cycles). The SOXA group underwent preoperative SOXA treatment ( 3 cycles; apatinib was discontinued in the third cycle, along with S-1) + surgery + postoperative SOXA treatment (3 cycles) + apatinib ( 3 cycles, apatinib can be continued after cycle 3 at the option of the investigator, overall treatment is up to 1 year of apatinib). The SOXAP group underwent preoperative SOXAP treatment (3 cycles; apatinib was discontinued in the third cycle, along with S-1) + surgery + postoperative SOXAP treatment (3 cycles) + apatinib combined with camrelizumab (3 cycles, apatinib in combination with camrelizumab can be continued after cycle 3 at the option of the investigator, overall treatment is up to 1 year of apatinib and up to 17 cycles of camrelizumab).

S-1 was administrated orally twice a day after meals, with the initial dose determined according to the body surface area: $80 \mathrm{mg}$ daily for participants with body surface area $<1.25 \mathrm{~m}^{2}, 100 \mathrm{mg}$ daily for those with body surface area $1.25-1.49 \mathrm{~m}^{2}$, and $120 \mathrm{mg}$ daily for those with body surface area $\geq 1.5 \mathrm{~m}^{2}$. After continuous dosing for 2 weeks, S-1 treatment was discontinued for 1 week. Oxaliplatin was administered intravenously at $130 \mathrm{mg} / \mathrm{m}^{2}$ on the first day of each cycle. Apatinib was orally administered once daily within 0.5 hours after a meal for 21 days at $500 \mathrm{mg}$ (SOXA group) or $250 \mathrm{mg}$ (SOXAP group). Camrelizumab was 
administered intravenously at $200 \mathrm{mg}$ (within 30-60 minutes) once every 3 weeks. Twenty-one days of treatment was considered one cycle. Standard D2 lymphadenectomy was recommended for radical surgery.

\section{Primary endpoint}

The primary endpoint was major pathological response (MPR) assessed by blinded independent review committee (BIRC), which was defined as the proportion of participants with a residual tumor in the primary tumor $<10 \%$ and Becker tumor regression grading 1 (1a or 1b) (19).

\section{Secondary endpoints}

The secondary endpoints were: (I) pathological complete response, which was defined as the proportion of participants with no residual tumor cells in the primary tumor and Becker stage 1a (19); (II) lymph node status after neoadjuvant therapy (ypN stage); (III) R0 resection rate; (IV) progressionfree survival (PFS), which was defined as the time from randomization to disease progression, recurrence, or death (whichever occurred first); (V) DFS, which was defined as the time from the first imaging assessment to disease recurrence or death (whichever occurred first); (VI) OS, which was defined as the time from randomization to all-cause death; and (VII) safety (including surgical and drug safety).

\section{Exploratory endpoint}

The exploratory endpoint was exploring and validating biomarkers as the predictors of efficacy with camrelizumab.

\section{Follow up and assessment}

The pathological response was assessed by a blinded independent review committee, according to the Becker criteria (19). The lymph node status and $\mathrm{R} 0$ resection rate were assessed according to the AJCC criteria, 8th edition (18). PFS, DFS, and OS were assessed by investigators, according to the Response Evaluation Criteria for Solid Tumors, version 1.1 (20). The participants were followed up every 3 months for the first year, and every 6 months thereafter. A second primary tumor and non-R0 resection are not considered in the assessment of PFS and DFS.

Surgical safety was assessed based on 30-day mortality, surgical complications (surgical site infection, anastomotic fistula, gastrointestinal bleeding, incision rupture, adhesive intestinal obstruction, biliary fistula, chylous fistula, fever [temperature $\geq 37.5^{\circ} \mathrm{C}$ ] of unknown origin), reoperation rate, length of hospital stay after surgery, and operation time. Drug safety was assessed with adverse events (AE), according to the National Cancer Institute Common Terminology Criteria for Adverse Event, version 5.0. Electronic case report forms were used for the data collection and data management.

\section{Sample size calculation}

Based on the literature, we assumed that the MPR in the SOXA, SOXAP, and SOX groups would be 36\%, 40\%, and $18 \%$, respectively $(3,21,22)$. Considering a $1: 1: 1$ randomization and one-sided significance level of 0.05 , we determined that 73 participants in each group would contribute $\geq 80 \%$ power to determine the superiority in the SOXA or SOXAP group versus the SOX group. Considering a $15 \%$ drop-out rate, 86 participants were required in each group, and thus 258 participants needed to be included in the present study.

\section{Statistical analysis}

The present study was a superiority trial that investigated the efficacy difference between the SOXAP and SOX groups. If there was a statistically significant difference between the SOXAP and SOX groups (one-sided $\mathrm{P}<0.05$ ), then the efficacy difference between the SOXA and SOX groups were investigated. If there was no statistically significant difference between the SOXAP and SOX groups, the comparison between the SOXA and SOX group were exploratory.

The intent-to-treat (ITT) population included all the participants randomized in the present study. The perprotocol set (PPS) included participants with no major deviation of the study protocol and the participants with protocol deviations that did not substantially influence the study results. The safety set (SS) included participants who were randomized and received at least one dose of the study drug. In the present study, the ITT population and PPS were used for the efficacy assessment, and the ITT population was the primary set. Safety was assessed with the SS.

The Cochran-Mantel-Haenszel test was used for comparisons of the primary and secondary endpoints (except for survival) between the SOX group and the other two groups. The Kaplan-Meier method was used to estimate the median survival and to plot the survival curve, the Brookmeyer-Crowley method was used to calculate the $95 \%$ confidence interval of the median survival, and the 


\section{Page 6 of 7}

stratified log-rank test was used to compare survival among the different groups. Logistic regression and forest plot were employed for the subgroup analysis of the primary endpoint.

\section{Discussion}

The aim of the present study was to compare the efficacy and safety of SOXA or SOXAP with SOX as the perioperative therapy for resectable, locally advanced gastric or GEJ adenocarcinoma. There are several advantages of the study design. First, we selected low-toxic chemotherapy drugs, antiVEGF targeting drugs, and immune checkpoint inhibitors in combination to improve patients' tolerance to the scheduled therapy. Resolve study demonstrated that SOX was suitable for the perioperative treatment (7). SOX combined with apatinib also showed safety in patients with locally advanced gastric adenocarcinoma (8). Moreover, apatinib plus camrelizumab had manageable safety in hepatocellular carcinoma, gastric or esophagogastric junction cancer (23). Any known potential adverse events to the study drugs were addressed in the protocol. Second, both regimens, SOXAP and SOXA, were tested in the present study, with standard SOX regimen as the positive control, which may successfully yield more treatment options for patients when the efficacy of the novel therapies is confirmed in the present study. Finally, preoperative staging was performed because all patients were pathologically diagnosed by gastroscopy and biopsy before randomization according to the investigator's judgment. Peritoneal dissemination and intra-abdominal metastasis were excluded by peritoneal cytological examination and exploratory laparoscopy based on the investigator's judgment.

In conclusion, the trial provides prospective multicenter data indicating the therapeutic potential of the perioperative SOXAP and SOXA regimens compared with the SOX regimen in patients with resectable, locally advanced gastric or GEJ adenocarcinoma in China. The results will contribute to optimal perioperative disease treatment.

\section{Acknowledgments}

We thank Fei Yuan and Benyan Zhang from the Department of Pathology of Ruijin Hospital for their suggestions on the pathological assessment in the study protocol (version 3.0, October 15, 2020).

Funding: The present study was supported by the Beijing Xisike Clinical Oncology Research Foundation (grant no. Y-HR2018-114).

\section{Zheng et al. Perioperative treatment for advanced gastric cancer}

\section{Footnote}

Reporting Checklist: The authors have completed the SPIRIT reporting checklist. Available at http://dx.doi.org/10.21037/ atm-20-7802

Conflicts of Interest: All authors have completed the ICMJE uniform disclosure form (available at http://dx.doi. org/10.21037/atm-20-7802). Dr. ZH and Dr. ZZ report other from Jiangsu Hengrui Medicine Co., Ltd., outside the submitted work; Dr. CL reports grants from Beijing Xisike Clinical Oncology Research Foundation, during the conduct of the study. The other authors have no conflicts of interest to declare.

Ethical Statement: The authors are accountable for all aspects of the work in ensuring that questions related to the accuracy or integrity of any part of the work are appropriately investigated and resolved. The present study is performed in accordance with the Declaration of Helsinki (as revised in 2013), Good Clinical Practice, and related laws. The protocol have been approved by the Ethics Committee at Ruijin Hospital affiliated with the School of Medicine at Shanghai Jiao Tong University (2019), (clinical ethics approval No. 212-3). All study participants provided signed informed consent prior to study commencement.

Open Access Statement: This is an Open Access article distributed in accordance with the Creative Commons Attribution-NonCommercial-NoDerivs 4.0 International License (CC BY-NC-ND 4.0), which permits the noncommercial replication and distribution of the article with the strict proviso that no changes or edits are made and the original work is properly cited (including links to both the formal publication through the relevant DOI and the license). See: https://creativecommons.org/licenses/by-nc-nd/4.0/.

\section{References}

1. Torre LA, Bray F, Siegel RL, et al. Global cancer statistics, 2012. CA Cancer J Clin 2015;65:87-108.

2. Wang $W$, Sun $Z$, Deng J, et al. Integration and analysis of associated data in surgical treatment of gastric cancer based on multicenter, high volume databases. Zhonghua Wei Chang Wai Ke Za Zhi 2016;19:179-85.

3. Cunningham D, Allum WH, Stenning SP, et al. Perioperative chemotherapy versus surgery alone for resectable gastroesophageal cancer. $\mathrm{N}$ Engl J Med 
2006;355:11-20.

4. Ychou M, Boige V, Pignon JP, et al. Perioperative chemotherapy compared with surgery alone for resectable gastroesophageal adenocarcinoma: an FNCLCC and FFCD multicenter phase III trial. J Clin Oncol 2011;29:1715-21.

5. NCCN Clinical Practice Guidelines in Oncology (NCCN Guidelines). Gastric cancer. Version 1.2020. Fort Washington: National Comprehensive Cancer Network; 2020.

6. Wang FH, Shen L, Li J, et al. The Chinese Society of Clinical Oncology (CSCO): clinical guidelines for the diagnosis and treatment of gastric cancer. Cancer Commun (Lond) 2019;39:10.

7. Ji J, Shen L, Li Z, et al. Perioperative chemotherapy of oxaliplatin combined with S-1 (SOX) versus postoperative chemotherapy of SOX or oxaliplatin with capecitabine (XELOX) in locally advanced gastric adenocarcinoma with D2 gastrectomy: A randomized phase III trial (RESOLVE trial). Ann Oncol 2019;30:v877.

8. Zheng Y, Yang X, Yan C, et al. Effect of apatinib plus neoadjuvant chemotherapy followed by resection on pathologic response in patients with locally advanced gastric adenocarcinoma: A single-arm, open-label, phase II trial. Eur J Cancer 2020;130:12-9.

9. Zitvogel L, Kroemer G. Targeting PD-1/PD-L1 interactions for cancer immunotherapy. Oncoimmunology 2012;1:1223-5.

10. Akinleye A, Rasool Z. Immune checkpoint inhibitors of PDL1 as cancer therapeutics. J Hematol Oncol 2019;12:92.

11. Kok VC. Current Understanding of the Mechanisms Underlying Immune Evasion From PD-1/PD-L1 Immune Checkpoint Blockade in Head and Neck Cancer. Front Oncol 2020;10:268.

12. Meyers DE, Bryan PM, Banerji S, et al. Targeting the PD-1/PD-L1 axis for the treatment of non-small-cell lung cancer. Curr Oncol 2018;25:e324-e34.

13. Seliger B. Basis of PD1/PD-L1 Therapies. J Clin Med 2019;8:2168.

14. Brar G, Shah MA. The role of pembrolizumab in the treatment of PD-L1 expressing gastric and gastroesophageal junction adenocarcinoma. Therap Adv Gastroenterol 2019;12:1756284819869767.

15. Akin Telli T, Bregni G, Camera S, et al. PD-1 and PDL1 inhibitors in oesophago-gastric cancers. Cancer Lett 2020;469:142-50.

16. Huang J, Mo H, Zhang W, et al. Promising efficacy of
SHR-1210, a novel anti-programmed cell death 1 antibody, in patients with advanced gastric and gastroesophageal junction cancer in China. Cancer 2019;125:742-9.

17. Shen L, Peng Z, Zhang YQ, et al. Camrelizumab combined with capecitabine and oxaliplatin followed by camrelizumab and apatinib as first-line therapy for advanced or metastatic gastric or gastroesophageal junction cancer: Updated results from a multicenter, open label phase II trial. J Clin Oncol 2019;37:abstr 4031.

18. Amin MB. AJCC Cancer Staging Manual, 8th Edition. Chicago: American College of Surgeons; 2018.

19. Becker K, Mueller JD, Schulmacher C, et al. Histomorphology and grading of regression in gastric carcinoma treated with neoadjuvant chemotherapy. Cancer 2003;98:1521-30.

20. Schwartz LH, Litiere S, de Vries E, et al. RECIST 1.1-Update and clarification: From the RECIST committee. Eur J Cancer 2016;62:132-7.

21. Cunningham D, Stenning SP, Smyth EC, et al. Perioperative chemotherapy with or without bevacizumab in operable oesophagogastric adenocarcinoma (UK Medical Research Council ST03): primary analysis results of a multicentre, open-label, randomised phase 2-3 trial. Lancet Oncol 2017;18:357-70.

22. Al-Batran SE, Hofheinz RD, Pauligk C, et al. Histopathological regression after neoadjuvant docetaxel, oxaliplatin, fluorouracil, and leucovorin versus epirubicin, cisplatin, and fluorouracil or capecitabine in patients with resectable gastric or gastro-oesophageal junction adenocarcinoma (FLOT4-AIO): results from the phase 2 part of a multicentre, open-label, randomised phase 2/3 trial. Lancet Oncol 2016;17:1697-708.

23. Xu J, Zhang Y, Jia R, et al. Anti-PD-1 Antibody SHR-1210 Combined with Apatinib for Advanced Hepatocellular Carcinoma, Gastric, or Esophagogastric Junction Cancer: An Open-label, Dose Escalation and Expansion Study. Clin Cancer Res 2019;25:515-23.

(English Language Editor: R. Scott)

Cite this article as: Zheng Y, Wang Z, Yan C, Yan M, Hou Z, Zheng R, Zhu Z, Li C. Protocol for a randomized controlled trial of perioperative S-1 plus oxaliplatin combined with apatinib and camrelizumab in patients with resectable, locally advanced gastric or esophagogastric junction adenocarcinoma. Ann Transl Med 2020;8(24):1684. doi: 10.21037/atm-20-7802 